\title{
Mitochondrial function in neuronal cells depends on p97/VCP/Cdc48-mediated quality control
}

\section{Lei Fang ${ }^{1}$, Charles Hemion ${ }^{1}$, Ana C. Pinho Ferreira Bento ${ }^{1}$, Claudia C. Bippes ${ }^{1}$, Josef Flammer $^{2}$ and Albert Neutzner ${ }^{1,2 *}$}

${ }^{1}$ Department of Biomedicine, University of Basel, Basel, Switzerland

${ }^{2}$ Department of Ophthalmology, University of Basel, Basel, Switzerland

\section{Edited by:}

Lawrence Rajendran, University of

Zurich, Switzerland

\section{Reviewed by:}

Hariharasubramanian Ramakrishnan,

State University of New York, USA

Krishna Vadodaria, Salk Institute for

Biological Science, USA

*Correspondence:

Albert Neutzner, Department of Biomedicine and Department of Ophthalmology, University of Basel, Hebelstrasse 20, 4031 Basel,

Switzerland

e-mail: albert.neutzner@unibas.ch
Maintaining mitochondrial function is essential for neuronal survival and offers protection against neurodegeneration. Ubiquitin-mediated, proteasome-dependent protein degradation in the form of outer mitochondrial membrane associated degradation (OMMAD) was shown to play roles in maintenance of mitochondria on the level of proteostasis, but also mitophagy and cell death. Recently, the AAA-ATPase p97/NCP/Cdc48 was recognized as part of OMMAD acting as retrotranslocase of ubiquitinated mitochondrial proteins for proteasomal degradation. Thus, p97 likely plays a major role in mitochondrial maintenance. Support for this notion comes from mitochondrial dysfunction associated with amyotrophic lateral sclerosis and hereditary inclusion body myopathy associated with Paget disease of bone and frontotemporal dementia (IBMPFD) caused by p97 mutation. Using SH-SY5Y cells stably expressing p97 or dominant-negative p97 ${ }^{\mathrm{OO}}$ treated with mitochondrial toxins rotenone, 6-OHDA, or A $\beta$-peptide as model for neuronal cells suffering from mitochondrial dysfunction, we found mitochondrial fragmentation under normal and stress conditions was significantly increased upon inactivation of p97. Furthermore, inactivation of p97 resulted in loss of mitochondrial membrane potential and increased production of reactive oxygen species (ROS). Under additional stress conditions, loss of mitochondrial membrane potential and increased ROS production was even more pronounced. Loss of mitochondrial fidelity upon inactivation of p97 was likely due to disturbed maintenance of mitochondrial proteostasis as the employed treatments neither induced mitophagy nor cell death. This was supported by the accumulation of oxidatively-damaged proteins on mitochondria in response to p97 inactivation. Dysfunction of p97 under normal and stress conditions in neuron-like cells severely impacts mitochondrial function, thus supporting for the first time a role for p97 as a major component of mitochondrial proteostasis.

Keywords: mitochondria, p97/VCP, neuronal cells, quality control

\section{INTRODUCTION}

Failing mitochondrial maintenance is at the heart of neurodegeneration and associated neuronal death (Karbowski and Neutzner, 2012; Kornmann, 2014). To prevent the untimely death of neuronal cells, mitochondria are kept healthy and in shape through the complex interplay of various molecular mechanisms aimed at repairing mitochondrial damage on the molecular level (Anand et al., 2013) or at removing damaged mitochondrial subunits from the cell (Youle and Narendra, 2011). A key component of mitochondrial maintenance is quality control of damaged, dysfunctional proteins through protein degradation (Neutzner et al., 2008; Escobar-Henriques and Langer, 2014). Owed to the complex architecture and the endosymbiotic nature of mitochondria, several protein degradation mechanisms are in place to maintain proteostasis in the various mitochondrial compartments (Tatsuta and Langer, 2008). Recently, we and others described roles for the ubiquitin-proteasome system in maintaining mitochondrial function and proteostatis.
E3 enzymes, namely MARCH5/MITOL (Karbowski et al., 2007; Nagashima et al., 2014), MAPL/MULAN (Braschi et al., 2009), IBRDC2 (Benard et al., 2010), RNF185 (Tang et al., 2011), and Parkin (Narendra et al., 2008) were found to localize to the mitochondrial outer membrane. Furthermore, ubiquitindependent protein degradation was shown to modulate mitochondrial morphology (Neutzner and Youle, 2005; Karbowski et al., 2007; Cohen et al., 2008; Leboucher et al., 2012) and impact mitophagy (Narendra et al., 2012). In addition, quality control of mitochondria-localized poly-Q (Sugiura et al., 2011), amyotrophic lateral sclerosis associated mSOD1 as well as Snitrosylated proteins (Benischke et al., 2014) is performed by the ubiquitin-proteasome system. Analogous to the endoplasmic reticulum (ER) which is quality controlled by ER associated degradation or ERAD (Ruggiano et al., 2014), mitochondria might be considered to be under control of outer mitochondrial membrane associated degradation (OMMAD, Neutzner et al., 2007). 
Quality control of proteins localized to a membrane-bound organelle by the cytosolic ubiquitin-proteasome system must involve the protein extraction from the organelle and retrotranslocation into the cytosol for degradation. The AAA-ATPase valosin containing protein $\mathrm{VCP} / \mathrm{p} 97 / \mathrm{Cdc} 48$ is the central component of this retrotranslocation machinery necessary for proteasomal degradation of organellar proteins (Meyer et al., 2012). Interestingly, p97 fulfils this function for ERAD (Wolf and Stolz, 2012) and OMMAD (Xu et al., 2011) alike. As such, p97 is an integral part of proteasomal quality control of ER-localized as well as mitochondrial proteins. While the role of $\mathrm{p} 97$ in maintaining ER proteostasis is extensively studied, the connection between p97 and mitochondrial health is less clear. However, p97 dysfunction was recently linked to some forms of amyotrophic lateral sclerosis and hereditary inclusion body myopathy associated with Paget disease of bone and frontotemporal dementia (IBMPFD) and a connection to failed mitochondrial quality control was suspected (Yamanaka et al., 2012).

To further define the role of $\mathrm{p} 97$ in mitochondrial maintenance especially in neuronal-like cells, we studied the influence of p97 inactivation on mitochondrial health and function in SH-SY5Y cells in comparison to known neurotoxic mitochondrial insults. We found that inactivation of p97 negatively impacts mitochondrial function in terms of membrane potential, reactive oxygen production, morphological changes, and accumulation of oxidized proteins comparably to treatment with the electron transport chain inhibitor rotenone, the neurotoxin 6-hydroxydopamine as well as Alzheimer's disease related A $\beta$ peptide. These findings support an important function for $\mathrm{p} 97$ in maintaining neuronal health through mitochondrial protein quality control and further strengthen the link between mitochondrial dysfunction and premature neuronal death.

\section{RESULTS}

\section{MITOCHONDRIAL FRAGMENTATION AS RESULT OF INACTIVATION OF p97}

Mitochondria fragment in response to mitochondrial insults such as the complex I inhibitor rotenone, the neurotoxic compound 6hydroxydopamine (6-OHDA) or the Alzheimer's-related peptide $\mathrm{A} \beta$. To assess whether $\mathrm{p} 97$ is involved in mitochondrial maintenance and stress protection in neuron-like cells, p97 function was blocked by overexpression of $\mathrm{p} 97^{\mathrm{QQ}}$, a dominant-negative version of p97, under conditions of mitochondrial insult. In order to induce low level mitochondrial insult, toxin concentration and time of insult was selected to minimize impact on cellular viability. To this end, SH-SY5Y neuroblastoma cells stably expressing p97 or dominant-negative $\mathrm{p} 97^{\mathrm{QQ}}$ under control of a tetracyclineinducible promoter were treated with tetracycline in the presence of rotenone, 6-OHDA, or $\mathrm{A} \beta$. As shown in Figure 1, expression of $\mathrm{p} 97^{\mathrm{QQ}}$ caused mitochondrial fragmentation in $57.3 \pm$ $5.9 \%$ compared to $5 \pm 2 \%$ in control cells expressing p97 and $7 \pm 1 \%$ in uninduced $\mathrm{p} 97^{\mathrm{QQ}}$ cells. Treatment of $\mathrm{p} 97$-expressing cells with the mitochondrial toxins rotenone, 6-OHDA or A $\beta$ resulted in mitochondrial fragmentation in $59.3 \pm 7.6 \%, 69 \pm$ $6.2 \%$, and $53.3 \pm 6.7 \%$, respectively. Interestingly, expression of $\mathrm{p} 97^{\mathrm{QQ}}$ in the presence of mitochondrial toxins had a strong additive effect and caused mitochondrial fragmentation in $92.3 \pm$
$3.1 \%$ (rotenone), $95 \pm 1.7 \%(6-\mathrm{OHDA})$, and $96.7 \pm 2.5 \%(\mathrm{~A} \beta)$ of SH-SY5Y cells. These data are consistent with a role for $\mathrm{p} 97$ in mitochondrial maintenance during normal as well as under mitochondrial stress conditions. To further explore this function of p97 on mitochondria in neuron-like cells, highly purified mitochondria were analyzed by western blotting for the presence of p97. As shown in Figure 1C, p97 was found in mitochondrial fractions from SH-SY5Y cells enriched in the mitochondrial marker cytochrome c, but devoid of the ER-marker calnexin. These data are consistent with p97 present on mitochondria and confirm previous observations of mitochondrial p97 (Xu et al., 2011).

\section{INACTIVATION OF p97 NEGATIVELY IMPACTS MITOCHONDRIAL MEMBRANE POTENTIAL AND INCREASES PRODUCTION OF REACTIVE OXYGEN SPECIES}

Mitochondrial membrane potential is a measure for mitochondrial health with a drop in membrane potential being a sign of mitochondrial dysfunction. Mitochondrial membrane potential was measured in neuron-like cells under conditions of mitochondrial insult during p97 inactivation to further assess the role of p97 in maintaining mitochondrial health. To this end, SH-SY5Y cells stably expressing $\mathrm{p} 97$ or $\mathrm{p} 97^{\mathrm{QQ}}$ were treated with rotenone, $6-\mathrm{OHDA}$, or $\mathrm{A} \beta$ or left untreated as control and mitochondrial membrane potential was measured using the membranepotential sensitive dye tetramethylrhodamine ethyl ester (TMRE). As shown in Figure 2A, ectopic expression of p97 did not impact mitochondrial membrane potential compared to control, while the potential was diminished to $80.6 \pm 6.0 \%$ of control by expression of $\mathrm{p} 97^{\mathrm{QQ}}$. Furthermore, treatment with mitochondrial toxins in addition to $\mathrm{p} 97^{\mathrm{QQ}}$ expression caused a further reduction of mitochondrial membrane potential to $60.9 \pm 8.3 \%$ (rotenone), $61.5 \pm 4.6 \%$ (6-OHDA), and $62.9 \pm 5.6 \%(\mathrm{~A} \beta)$. Pharmacological inhibition of p97 was employed to confirm the observed impact of p97 inactivation on mitochondrial membrane potential. To this end, SH-SY5Y cells were treated with the specific p97 inhibitor DBeQ or vehicle (DMSO) and flow cytometric analysis of TMRE fluorescence was performed. As shown in Figure 2B, DBeQ-dependent inhibition of p97 caused a reduction of TMRE fluorescence to $74.7 \pm 11.5$ of control cells $(p=0.072)$ comparable to the drop of mitochondrial membrane potential following expression of $\mathrm{p} 97^{\mathrm{QQ}}$.

In addition to mitochondrial depolarization, increased production of reactive oxygen species (ROS) is a hallmark of failing mitochondrial maintenance and subsequent dysfunction. To further analyse the role of p97 in mitochondrial maintenance in neuron-like cells cells, ROS production was measured under mitochondrial stress conditions in the presence or absence of $\mathrm{p} 97$ function. Again, SH-SY5Y cells stably containing tetracyclineinducible $\mathrm{p} 97$ or $\mathrm{p} 97^{\mathrm{QQ}}$ were induced with tetracycline, treated with rotenone, 6-OHDA, or $\mathrm{A} \beta$ or left untreated as control, and mitochondrial ROS levels were analyzed by flow cytometry using the ROS-sensitive dye MitoSox. While ectopic expression of p97 had only a minor influence on ROS levels (114.9 $\pm 8.7 \%)$ compared to uninduced control cells (Figure 2C), expression of p97 ${ }^{\mathrm{QQ}}$ alone caused ROS levels to increase to $212.7 \pm 18.7 \%$. Additional treatment of p97 expressing cells with mitochondrial 


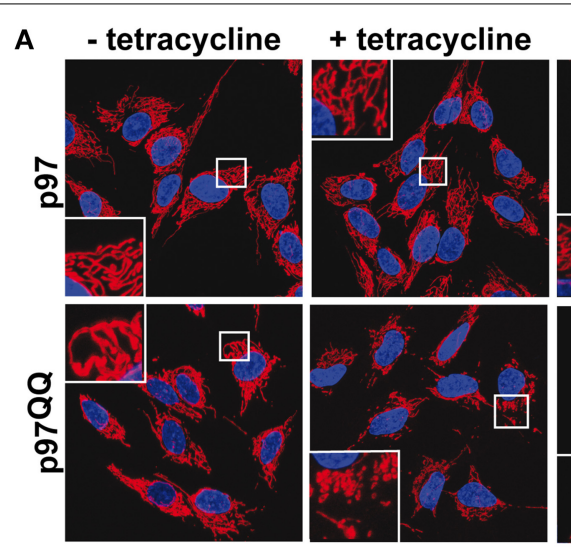

B

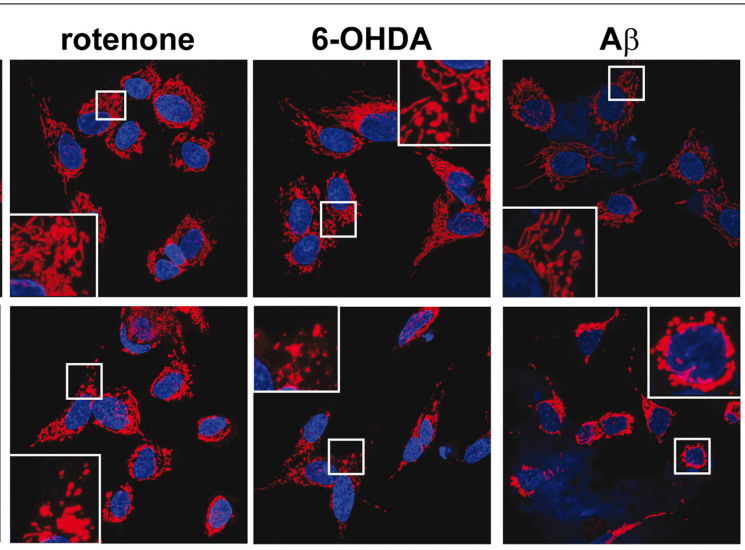

C
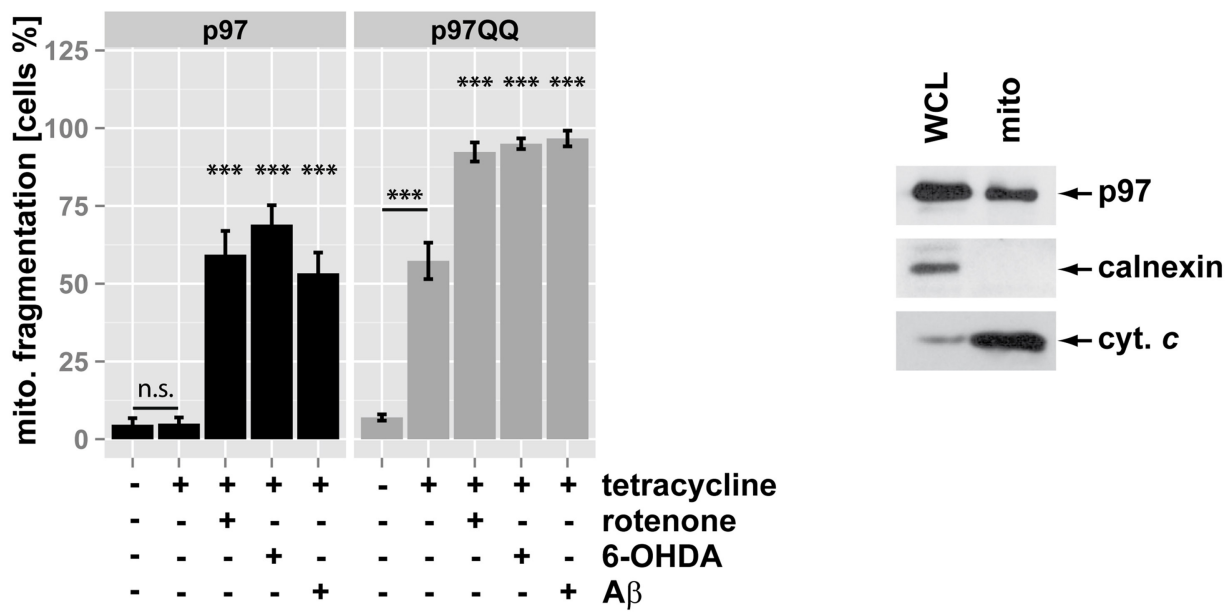

FIGURE 1 | Inactivation of p97 increased mitochondrial fragmentation under stress. (A) SH-SY5Y cells stably expressing p97 or

dominant-negative $\mathrm{p} 9700$ under control of the Tet-On promoter were induced with tetracycline for $16 \mathrm{~h}$ or left uninduced and treated with $5 \mu \mathrm{M}$ rotenone, $75 \mu \mathrm{M} 6 \mathrm{OHDA}$, or $50 \mu \mathrm{M}$ A $\beta$ for $6 \mathrm{~h}$. Cells were fixed and stained using anti-cytochrome $c$ antibody and Alexa546-conjugated secondary antibody. Shown are representative pictures from three independent experiments. (B) Fragmentation of the mitochondrial network in cells from (A) was quantified by visual examination. Shown is the average of three independent experiments. Statistical analysis was performed using pair-wise $t$-tests with $p$-value adjustment according to Holm. Statistical significance is marked with n.s. for not significant, ${ }^{* * *} p<0.001$. (C) The presence of $p 97$ on mitochondria was analyzed by western blotting using anti-p97 antibodies following purification of mitochondria using anti-TOMM22 magnetic beads. The purity of mitochondrial fractions was assessed by detecting the ER marker calnexin and the mitochondrial marker cytochrome c (cyt. c). Shown is one representative out of three independent experiments. toxins further increased ROS levels to $158 \pm 8.3 \%$ (rotenone), $167.7 \pm 9 \%(6-\mathrm{OHDA})$, and $294.3 \pm 14.1 \%(\mathrm{~A} \beta)$. In response to $\mathrm{p} 97$ inactivation under mitochondrial stress conditions ROS levels further increased to $290.4 \pm 55.6 \%$ (rotenone), $360.9 \pm$ $58.9 \%$ (6-OHDA), and $611.5 \pm 129.1 \%(\mathrm{~A} \beta)$. As above, pharmacological inhibition was used to confirm the impact of $\mathrm{p} 97$ on mitochondrial ROS production (Figure 2D). Treatment with the p97 inhibitor DBeQ caused a highly significant $1.8 \pm 0.2$ fold increase in ROS production compared to vehicle treated control cells again confirming the observed elevated ROS levels following expression of $\mathrm{p} 97^{\mathrm{QQ}}$. The observed increase of ROS production following $\mathrm{p} 97$ inactivation and the significant additive effect on ROS production of inactive p97 during mitochondrial stress strongly support a crucial role for p97-dependent mitochondrial maintenance under normal as well as stress conditions.

\section{INACTIVATION OF p97 IMPAIRS MITOCHONDRIAL MAINTENANCE BELOW THE MITOPHAGIC THRESHOLD}

Recently, p97 was implicated in the execution of mitophagy (Tanaka et al., 2010). To assess whether the observed mitochondrial dysfunction under the employed stress conditions and p97 inactivation are linked to blocked mitophagy or might be attributed to other roles of p97, Parkin translocation to mitochondria (Narendra et al., 2008) as a marker for mitophagic induction was measured. To this end, SH-SY5Y cells stably expressing p97 or p97 ${ }^{\mathrm{QQ}}$ were transfected with an expression construct for YFP-tagged Parkin, induced with tetracycline and exposed to mitochondrial insults by treating with rotenone, 6-OHDA, or $\mathrm{A} \beta$. Treatment with the protonophore (3-Chlorophenyl)hydrazonomalononitrile (CCCP) capable of complete mitochondrial depolarization and subsequent Parkin 

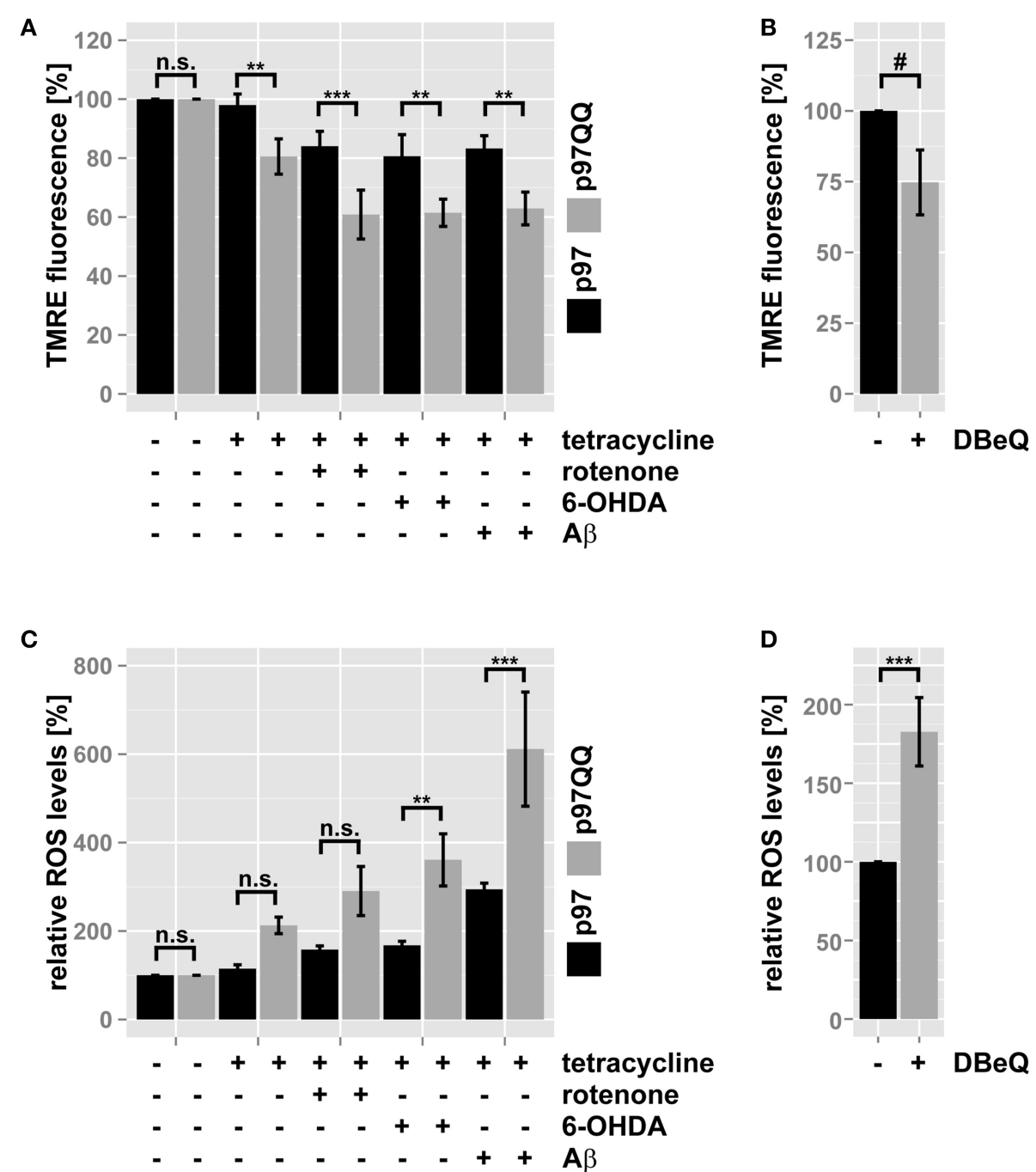

FIGURE 2 | Inactivation of p97 impairs mitochondrial function during neurotoxic stress. (A) SH-SY5Y cells stably expressing p97 or dominant-negative $\mathrm{p} 97 \mathrm{QQ}$ under control of the Tet-On promoter were induced with tetracycline for $2 \mathrm{~h}$ or left uninduced and treated with $5 \mu \mathrm{M}$ rotenone, $75 \mu \mathrm{M} 6 \mathrm{OHDA}$, or $50 \mu \mathrm{M} A \beta$ for an additional $6 \mathrm{~h}$. Cells were stained with the mitochondrial membrane sensitive dye TMRE and analyzed by flow cytometry. (B) SH-SY5Y cells were treated with the p97 inhibitor $\mathrm{DBeO}$ and mitochondrial membrane potential was measured by flow cytometric analysis of TMRE fluorescence. (C) Cells treated as in (A) were stained with the ROS-sensitive dye MitoSox and mitochondrial ROS generation was measured using flow cytometry. (D) SH-SY5Y cells were treated with the p97 inhibitor $\mathrm{DBeQ}$ and mitochondrial ROS generation was measured by flow cytometric analysis of MitoSox fluorescence. Statistical analysis was performed using pair-wise $t$-tests with Holm p-value adjustment for $(\mathbf{A}, \mathbf{C})$, and a general linear model (SPSS) for (B,D). Statistical significance is marked with n.s. for not significant, ${ }^{\#} p<0.1,{ }^{* *} p<0.01,{ }^{* * *} p<0.001$. translocation served as control. As shown in Figures 3A,B, while treatment with CCCP induced Parkin translocation in almost all cells, neither expression of $\mathrm{p} 97$ or $\mathrm{p} 97^{\mathrm{QQ}}$, nor neurotoxin treatment at the used concentrations caused significant $(<4 \%)$ translocation of Parkin to mitochondria. Consistent with this finding, the employed concentrations of rotenone, 6-OHDA, and $\mathrm{A} \beta$ also did not lead to cell death as measured by flow cytometry (Figure 3C), neither in the presence of ectopic p97 nor following expression of $\mathrm{p} 97^{\mathrm{QQ}}$. Thus, while the employed treatments with neurotoxic substances degraded mitochondrial function, the threshold for mitophagic induction as well as cell death was not reached. Therefore, the observed role for p97 in mitochondrial maintenance under the stress conditions employed by us is also likely on the protein degradation level rather than on the mitophagic or cell death level.

\section{p97 IS INVOLVED IN CLEARING OXIDATIVELY-DAMAGED PROTEINS FROM MITOCHONDRIA}

To further support this notion, the ubiquitin- and p97-mediated proteasome-dependent turnover of oxidized mitochondrial proteins under mitochondrial stress conditions was measured. In SH-SY5Y cells ectopically expressing p97, treatment with neither rotenone, nor 6-OHDA or $\mathrm{A} \beta$ significantly increased levels of oxidized proteins compared to untreated control cells (Figure 4, 
A
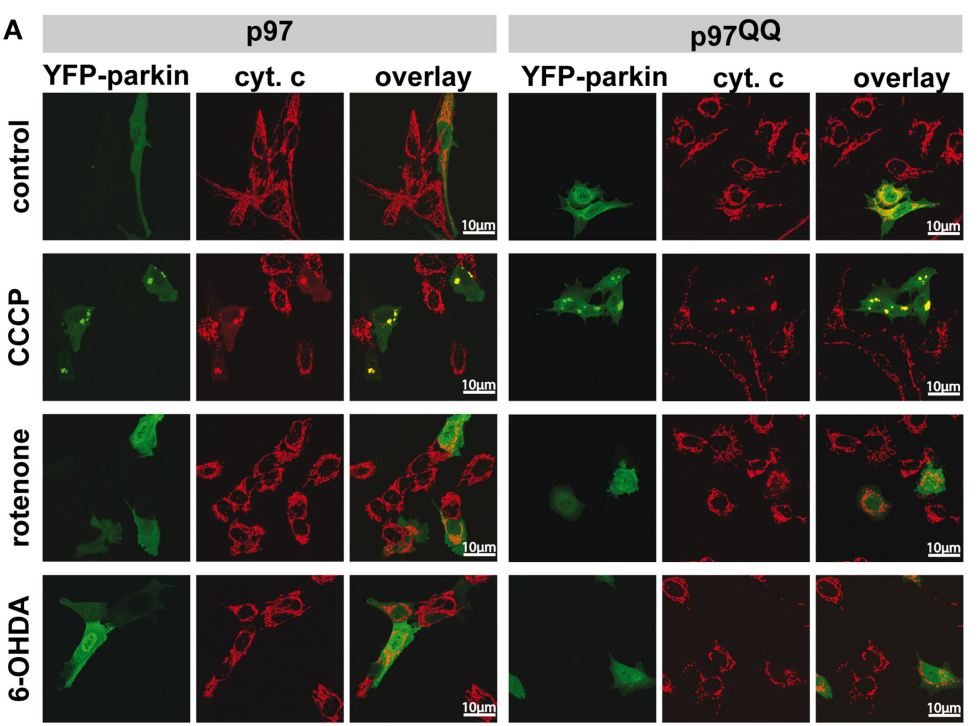
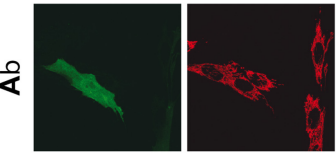

B

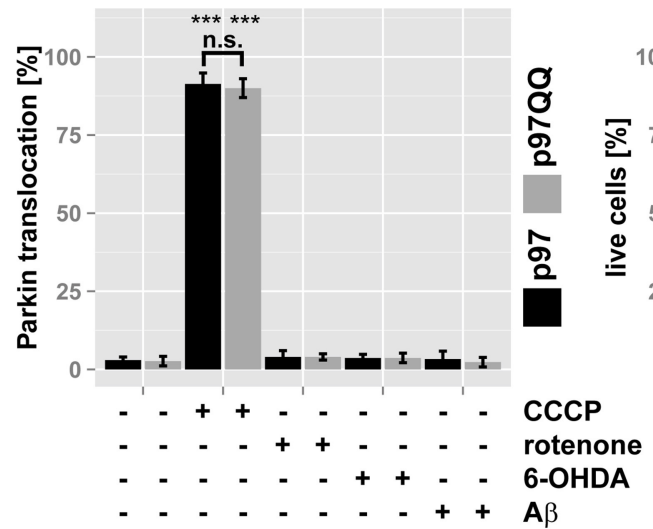

FIGURE 3 | p97 is involved in mitochondrial maintenance at damage levels below the thresholds for mitophagy and cell death. (A) SH-SY5Y cells stably expressing p97 or dominant-negative p9700 under control of the Tet-On promoter were transfected with an expression construct for YFP-tagged Parkin, induced with tetracycline for $2 \mathrm{~h}$ or left uninduced and treated with $5 \mu \mathrm{M}$ rotenone, $75 \mu \mathrm{M} 6$ OHDA, or $50 \mu \mathrm{M} \mathrm{A} \beta$ for an additional $6 \mathrm{~h}$. Cells were fixed, stained for the mitochondrial marker cytochrome c (cyt. c) and Parkin translocation from the cytosol to mitochondria was visually

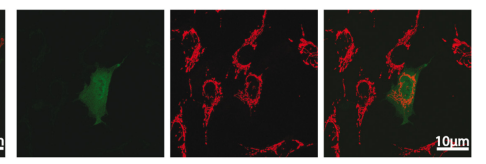

C

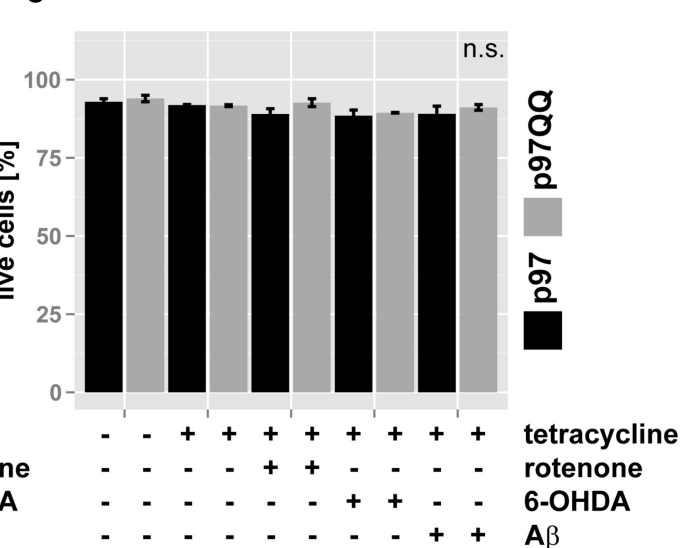

analyzed using fluorescence microscopy. Shown are representative images of three independent experiments. (B) Shown is a quantification of Parkin translocation from the cytosol to mitochondria from (A). (C) Cells treated as in (A) were stained with the cell impermeable dye 4',6-diamidino-2-phenylindole (DAPI) and the percentage of dead cells was determined by flow cytometry. Statistical analysis was performed using pair-wise $t$-tests with $p$-value adjustment according to Holm. Statistical significance is marked with *** $p<0.001$. In (C) no statistical significance was observed. for representative western blots see Supplementary Figure S1). Interestingly, in $\mathrm{p} 97^{\mathrm{QQ}}$ expressing cells, levels of oxidized mitochondrial proteins were significantly increased $(p<0.001)$ compared to p97 expressing cells confirming a function for p97 in the removal of oxidatively-damaged mitochondrial proteins. Furthermore, levels of oxidized proteins significantly increased in $\mathrm{p} 97^{\mathrm{QQ}}$ expressing cells under mitochondrial stress conditions due to rotenone, 6-OHDA, and $\mathrm{A} \beta$ treatment compared to $\mathrm{p} 97^{\mathrm{QQ}}$ control cells. Again, these data are consistent with the involvement of p97 in the degradation of damaged proteins to maintain mitochondrial function in neuron-like cells under mitochondrial stress conditions below the threshold of mitophagic induction or cell death.

\section{DISCUSSION}

Mechanisms of mitochondrial maintenance act on the cellular level by removing complete dysfunctional mitochondrial networks through programmed cell death. On the organellar level 


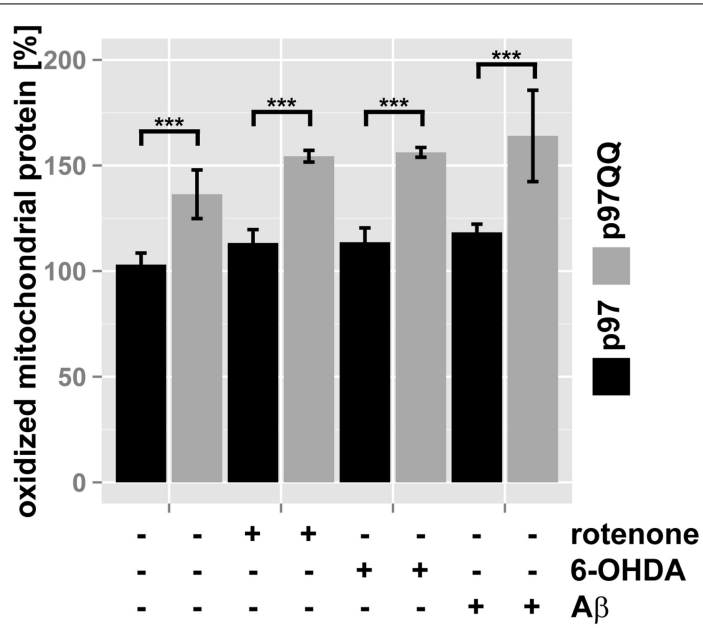

FIGURE 4 | Clearance of oxidatively-damaged mitochondrial proteins is impaired following inactivation of p97. SH-SY5Y cells stably expressing p97 or dominant-negative p970Q under control of the Tet-On promoter were induced with tetracycline for $2 \mathrm{~h}$ or left uninduced and treated with $5 \mu \mathrm{M}$ rotenone, $75 \mu \mathrm{M} 6 \mathrm{OHDA}$, or $50 \mu \mathrm{M} \mathrm{A} \beta$ for an additional $6 \mathrm{~h}$. Mitochondria were isolated using anti-TOMM22 magnetic beads resulting in highly purified mitochondria. Protein carbonylation as measure for oxidative damage was determined by infrared laser-based quantitative western blotting following derivatization with 2,4-Dinitrophenylhydrazine (DNPH) and detection using anti-DNP antibodies. Shown is the average of three independent experiments. Please see Supplementary Figure S1 for representative western blot images. Statistical analysis was performed using pair-wise $t$-tests with $p$-value adjustment according to Holm. Comparisons shown are tetracycline-induced, untreated cells vs. tetracycline-induced, treated cells. Statistical significance is marked with n.s. for not significant, ${ }^{* * *} p<0.001$.

they act through mitophagic degradation of dysfunctional mitochondrial subunits and finally on the molecular level by repairing and/or removing damaged mitochondrial components such as DNA, lipids or proteins (Tatsuta and Langer, 2008). While programmed cell death is an effective means to rid the body of damaged and, due to their excessive ROS production, potentially harmful mitochondrial networks, in post-mitotic neurons excessive apoptotic cell death is equivalent to neurodegeneration (Radi et al., 2014). Thus, mitophagy and degradation of damaged proteins are likely the first lines of defense against mitochondrial dysfunction in neurons as neuronal death is avoided. Which mechanism, mitophagy or protein degradation is more prevalent in neurons under normal conditions remains unclear. However, it is conceivable that the removal of individual components such as damaged proteins from otherwise functional mitochondria might be preferable to the destruction of whole mitochondrial subunits. Especially under conditions of everyday stress, with slowly accumulating, low overall mitochondrial damage, keeping mitochondrial damage below the mitophagic threshold through the constant removal of damaged proteins is likely to prevent neuronal damage.

Using known mitochondrial toxins such as rotenone, 6OHDA, and $A \beta$ at concentrations and treatment times below the threshold of cell death and mitophagic induction, we intended to model the above mentioned low level stress and evaluate the importance of p97 and associated protein degradation as well as its influence on mitochondrial fidelity in neuronal cells. Interestingly, inactivation of p97 alone-without additional exogenous stress-for as little as $8 \mathrm{~h}$ negatively influenced mitochondrial morphology, membrane potential and ROS production. Although $\mathrm{p} 97$ has many cellular functions and pleiotropic effects of $\mathrm{p} 97$ inactivation have to be taken into account, these observations are consistent with a direct role for p97 in mitochondrial maintenance under normal conditions in the absence of external mitochondrial stress. This notion is further supported by the accumulation of oxidatively-damaged mitochondrial proteins following p97 inactivation. Even under normal conditions without exogenous mitochondrial stress, a considerable amount of oxidized protein is present in mitochondria and significantly accumulates as consequence of a short period of p97 inactivation. Thus, in the absence of any detectable mitophagic activity, continuous turnover of damaged mitochondrial proteins occurs in a p97-dependent manner. As p97 is an ubiquitin-dependent chaperone, this is consistent with constantly ongoing mitochondrial maintenance in neuronal cells through the ubiquitin-proteasome system under normal, unstressed conditions. Consistent with this finding, we also found p97 to be present in mitochondrial fractions under normal conditions, and recently we also connected p97 and oxidatively-damaged proteins to OMMAD, the ubiquitin-dependent, proteasomemediated degradation mitochondrial proteins (Hemion et al., 2014). Further support to this notion is lent by the effect of p97 inactivation under exogenous stress conditions below the mitophagic threshold. Concurrent stress and p97 inactivation had additive effects on mitochondrial fragmentation, membrane potential, ROS production as well as the accumulation of oxidatively-damaged mitochondrial proteins. These observations again support a function for p97 in dealing with mitochondrial damage to keep dysfunction of mitochondria below the threshold above which mitophagic degradation or even cell death occurs. Thus, we propose that constant repair of mitochondrial damage by the ubiquitin-proteasome system in a p97-dependent manner even under conditions of no or low exogenous mitochondrial stress is critically important for maintaining mitochondrial function under normal conditions. Mitophagic clearance on the other hand might be responsible for maintaining mitochondrial function in response to more drastic insults likely not encountered under normal conditions. Taken together, p97-mediated mitochondrial proteostasis is likely an important mechanism to prevent mitochondrial dysfunction as result of slowly accruing mitochondrial damage under normal conditions and to keep associated neurodegenerative processes at bay.

\section{MATERIALS AND METHODS DNA CONSTRUCTS}

To generate a vector for the one-step generation of stable, tetracycline-inducible human cell lines, the CMV promoter in the AAVS1 donor cloning vector DC-DON-SH01 (GenomeTALER $^{\text {TM }}$ human AAVS1 safe harbor gene knock-in kit, GeneCopoeia) was replaced by the hybrid CMV/Tet-On promoter originating from pcDNA5/FRT/TO (Invitrogen) with MluI/PmeI. Afterwards, a stuffer sequence with $5^{\prime}$ PmeI site 
followed by EcoRV site and $3^{\prime}$ BstBI site was inserted using PmeI, $B s t \mathrm{BI}$ to generate $\mathrm{pAN} 2066$. To enable tetracycline regulation of the construct, the GFP coding region in pAN2066 was replaced with the coding sequence of the tetracycline repressor. To this end, the bGH poly-A-Ef1 $\alpha-G F P$ fragment was amplified from DC-DON-SH01 using ATTCGACTCGAGTTCGAATTTAA ATCGGATCCCT and ATTCGAGATATCGATCCGGTGGAG CCGGG and cloned into pBluescript SK (+) with XhoI/EcoRV to create pAN2067. Next, GFP was replaced by TetR amplified using PCR from pcDNA6/TR (Invitrogen) with ATTCGAAAGCTT GTGAGTTTGGGGACCCTTG and ATTCGAGATATCGCAT AAGATCTGAATTCCCGGGA and inserted HindIII/EcoRV to generate pAN2070. Fragment bGH poly-A-Ef1 $\alpha-T e t R$ was released from pAN2070 with $B s t \mathrm{BI} / E c o \mathrm{RV}$ and transferred to pAN2066 cut with BstBI/NruI to obtain pAN2071. Then, p97 or p97 QQ (gift from S. Fang) was amplified by PCR using GACT CGGATATCATGGCTTCTGGAGCCGATTCAA and TGTA ACAACGTTTTAGCCATACAGGTCATCATCATCATT and cloned EcoRV/BstBI into pAN2071.

\section{Cell culture and generation of cell lines}

$\mathrm{SH}-\mathrm{SY} 5 \mathrm{Y}$ cells were cultured in $5 \% \mathrm{CO}_{2}$ incubator at $37^{\circ} \mathrm{C}$ in high glucose DMEM (Sigma, D6546) containing 15\% Tet System Approved FBS (Clontech, 631106), supplemented with $2 \mathrm{mM} \mathrm{L-}$ glutamine (Sigma, G5713), and $1 \mathrm{mM}$ sodium pyruvate (Sigma, G7513). To generate stably transfected SH-SY5Y cells expressing $\mathrm{p} 97$ or $\mathrm{p} 97^{\mathrm{QQ}}$ under control of the Tet-On promoter, cells were transfected using Effectene (Qiagen, 301425) according to manufacturer's recommendations with two TALEN constructs for the PPP1R2C or AAVS1 "safe harbor" locus and an expression construct containing $\mathrm{p} 97$ or $\mathrm{p} 97^{\mathrm{QQ}}$ under control of the TetOn promoter as well as a puromycin resistance gene to enable selection. For stably transfected cells, $0.75 \mu \mathrm{g} / \mathrm{ml}$ puromycin was added to maintain selection (Invivogen, ant-pr-1). Expression of p97 or $\mathrm{p} 97^{\mathrm{QQ}}$ was induced by treatment with $1 \mu \mathrm{g} / \mathrm{ml}$ tetracycline (Roth, Hp63.1). Mitochondrial stress was induced by treatment with $5 \mu \mathrm{M}$ rotenone (Sigma, R8857), $75 \mu \mathrm{M}$ 6-hydroxdopamine (Sigma, H8523) or $50 \mu \mathrm{M}$ amyloid- $\beta$ protein fragment 25-35 (Sigma, A4559). For pharmacological inhibition of p97 function, SH-SY5Y cells were treated with the p97-inhibitor DBeQ (Sigma, SML0031) at $2.5 \mu \mathrm{M}$ for $20 \mathrm{~h}$ before further analysis (Chou et al., 2011).

\section{FLOW CYTOMETRY}

Flow cytometry was performed using a CyAn ADP Analyzer (Beckman Coulter). SH-SY5Y cells grown in 6 well cell culture plates (Sarstedt) and induced and/or treated as indicated were co-stained with DAPI and either $10 \mathrm{nM}$ TMRE (Invitrogen) for $30 \mathrm{~min}$ at $37^{\circ} \mathrm{C}$, or $5 \mu \mathrm{M}$ MitoSOX ${ }^{\mathrm{TM}}$ (Invitrogen) for $10 \mathrm{~min}$ at $37^{\circ} \mathrm{C}$. Cells were harvested, washed twice in PBS and resuspended in $1 \mathrm{ml}$ PBS containing $0.5 \%(\mathrm{w} / \mathrm{v})$ BSA and $50 \mu \mathrm{M}$ EDTA. Flow cytometry was performed immediately afterwards. Data analysis was performed using FlowJo v.10. Sequential gating was performed as follows (with identical gates used for each experiment): cells were gated for using logarithmic forward/sideward scatter axes; doublet discrimination was performed using forward scatter area/forward scatter followed by pulse width/forward scatter dot plots. Dead cells were excluded in DAPI/forward scatter dot plots.

\section{ISOLATION OF MITOCHONDRIA}

The human mitochondria isolation kit (Miltenyi Biotec, 130094-532) was used according to manufacturer's instructions. Briefly, cells were harvested and either directly processed or stored overnight in liquid nitrogen. All following steps were performed on ice with pre-cooled buffers. Cells were resuspended in $800 \mu \mathrm{l}$ lysis buffer supplemented with protease inhibitors $(1 \mu \mathrm{g} / \mathrm{ml}$ pepstatin; $1 \mu \mathrm{g} / \mathrm{ml}$ leupeptin; $1 \mathrm{mM}$ PMSF) and $50 \mu \mathrm{M}$ EDTA. Cells were passed 15 times through a 25 gage needle. Nine milliliter separation buffer and $50 \mu \mathrm{l}$ Anti-TOMM22 MicroBeads were added to the cell homogenate before rotating the suspensions for $1 \mathrm{~h}$ at $4^{\circ} \mathrm{C}$. Magnetic separation was performed using a MACS Separator. Purified mitochondria were immediately lysed in RIPA buffer (Thermo Scientific) supplemented with protease inhibitors ( $1 \mu \mathrm{g} / \mathrm{ml}$ pepstatin; $1 \mu \mathrm{g} / \mathrm{ml}$ leupeptin; $1 \mathrm{mM}$ PMSF) and $50 \mu \mathrm{M}$ EDTA and subjected to five $10 \mathrm{~s}$ intervals of sonication at $10 \mathrm{kHz}$. Total protein content was measured using the Pierce BCA protein assay kit (Thermo Scientific) and was immediately followed by DNPH labeling.

\section{LABELING WITH DNPH}

Labeling with DNPH was performed according to Wehr and Levine (2013) with minor alterations. Briefly, $60 \mu \mathrm{g}$ of total protein in $20 \mu \mathrm{l}$ total volume was added to $20 \mu \mathrm{l} 12 \%$ sodium dodecyl sulfate (SDS). $40 \mu \mathrm{l}$ of $20 \mathrm{mM} \mathrm{DNPH}$ in $2 \mathrm{M} \mathrm{HCl}$ were added, samples were briefly mixed and incubated for $15 \mathrm{~min}$. To control for DNPH-reactive protein carbonyls, samples were reacted with $2 \mathrm{M} \mathrm{HCl}$ lacking DNPH. $30 \mu \mathrm{l}$ of $2 \mathrm{M}$ Tris base, $30 \%$ glycerol and a final concentration of $50 \mathrm{mM}$ 1,4-Dithiothreit were added to stop the reaction. Samples were briefly mixed again and immediately used for SDS PAGE.

\section{WESTERN BLOT}

DNPH-labeled protein lysates were resolved by SDS PAGE $(5 \mu \mathrm{g}$ total protein per lane were loaded) and blotted onto nitrocellulose membrane (Whatman). Fast Green FCF (Sigma Aldrich, F7252) was used to evaluate total protein loaded per lane as follows: nitrocellulose membranes were stained for $10 \mathrm{~min}$ in Fast Green FCF staining solution (0.001\% FCF, 30\% methanol, 7\% acetic acid), followed by $10 \mathrm{~min}$ in destaining solution $(30 \%$ methanol, 7\% acetic acid) and then $10 \mathrm{~min}$ in water. An infrared laser scanner (LiCor) was used for detection of FCF. Following Fast Green FCF, membranes were blocked for $1 \mathrm{~h}$ in $3 \%(\mathrm{w} / \mathrm{v})$ Top Block (Lubio science, TB232010) in PBS-Tween 20 (0.05\%) and immune-detection using polyclonal rabbit anti-DNP antibodies (D9656 Sigma, 1:2000) and polyclonal goat anti-rabbit Dylight 800 (35521 Pierce, 1:6000) was performed and detected using an infrared laser scanner (LiCor) to obtain quantitative measurement. Each experiment was performed three times independently and each measurement was performed in triplicates. Image analysis was performed using ImageJ (Schneider et al., 2012). For the analysis of mitochondrial fractions by western blotting mouse anti-p97 (10R-P104a Fitzgerald, 1:1000), mouse anti-cytochrome $c$ (556433 Pharmingen, 1:2000), and 
rabbit anti-calnexin (ab75801 abcam, 1:2000) were used and detected using anti-mouse HRP or anti-rabbit HRP (Thermo Scientific) and ECL Plus chemiluminescent reagent (1896327 Thermo Scientific).

\section{MICROCOPY}

Cells were seeded in 6-well plates onto glass slides at $1 \times 10^{4}$ cells/well in $2 \mathrm{ml}$ culture medium. Samples were fixed using methanol-free electron microscopy grade $4 \%$ paraformaldehyde in PBS for $15 \mathrm{~min}$ at RT, permeabilized for $15 \mathrm{~min}$ at RT using $0.15 \%$ Trixon X-100 in PBS and blocked for $1 \mathrm{~h}$ in $10 \% \mathrm{BSA}(\mathrm{w} / \mathrm{v})$ in PBS. To visualize mitochondria, samples were then incubated with mouse anti-cytochrome $c$ antibody (BD Biosciences 556432, 1:1000) overnight at $4^{\circ} \mathrm{C}$ and Alexa546-conjugated anti-mouse antibodies (Invitrogen A11003, 1:500) for $1 \mathrm{~h}$ at RT. Nuclei were stained by incubation with DAPI (Invitrogen, D1306, 1:1000) for 5 min right after Alexa546 incubation. Samples were mounted in mounting medium (Vectashield, H1000) and observed using a confocal microscope (Zeiss LSM Meta710, $63 \times / 1.4$ objective). Mitochondria fragmentation and Parkin-to-mitochondria translocation were assessed visually and quantified as percentage of control. The extend of mitochondrial fragmentation was judged based on comparison to untreated control cells with mitochondrial networks scored as fragmented if most mitochondria in a cell did no longer exhibit an elongated phenotype. All experiments were performed independently in triplicates.

\section{STATISTICAL ANALYSIS}

Statistical significance of differences was assessed using pair-wise $t$-tests with the adjustment for multiple comparisons according to Holmes as implemented in R (R Core Team, 2013). Significance is indicated with n.s. $p>0.05,{ }^{*}$ for $p<0.05,{ }^{* *}$ for $p<0.01$, and *** for $p<0.001$.

\section{AUTHOR CONTRIBUTIONS}

Lei Fang, Charles Hemion, Claudia C. Bippes performed the experiments. Albert Neutzner conceived the study. Albert Neutzner, Lei Fang planned the experiments. Josef Flammer provided intellectual input. Josef Flammer, Lei Fang, Charles Hemion, Claudia C. Bippes, Albert Neutzner wrote the manuscript.

\section{ACKNOWLEDGMENTS}

This work was supported a grant of the Swiss National Foundation (31003A_146118) to Albert Neutzner. The authors want to acknowledge the FACS and light microscopy core facilities of the Department for Biomedicine, University Basel for their support.

\section{SUPPLEMENTARY MATERIAL}

The Supplementary Material for this article can be found online at: http://www.frontiersin.org/journal/10.3389/fncel.2015. 00016/abstract

\section{REFERENCES}

Anand, R., Langer, T., and Baker, M. J. (2013). Proteolytic control of mitochondrial function and morphogenesis. Biochim. Biophys. Acta 1833, 195-204. doi: 10.1016/j.bbamcr.2012.06.025
Benard, G., Neutzner, A., Peng, G., Wang, C., Livak, F., Youle, R. J., et al. (2010). IBRDC2, an IBR-type E3 ubiquitin ligase, is a regulatory factor for Bax and apoptosis activation. EMBO J. 29, 1458-1471. doi: 10.1038/emboj. 2010.39

Benischke, A. S., Hemion, C., Flammer, J., and Neutzner, A. (2014). Proteasomemediated quality control of S-nitrosylated mitochondrial proteins. Mitochondrion. 17, 182-186. doi: 10.1016/j.mito.2014.04.001

Braschi, E., Zunino, R., and McBride, H. M. (2009). MAPL is a new mitochondrial SUMO E3 ligase that regulates mitochondrial fission. EMBO Rep. 10, 748-754. doi: 10.1038/embor.2009.86

Chou, T. F., Brown, S. J., Minond, D., Nordin, B. E., Li, K., Jones, A. C., et al. (2011). Reversible inhibitor of p97, DBeQ, impairs both ubiquitin-dependent and autophagic protein clearance pathways. Proc. Natl. Acad. Sci. U.S.A. 108, 4834-4839. doi: 10.1073/pnas.1015312108

Cohen, M. M., Leboucher, G. P., Livnat-Levanon, N., Glickman, M. H., and Weissman, A. M. (2008). Ubiquitin-proteasome-dependent degradation of a mitofusin, a critical regulator of mitochondrial fusion. Mol. Biol. Cell 19, 2457-2464. doi: 10.1091/mbc.E08-02-0227

Escobar-Henriques, M., and Langer, T. (2014). Dynamic survey of mitochondria by ubiquitin. EMBO Rep. 15, 231-243. doi: 10.1002/embr.201338225

Hemion, C., Flammer, J., and Neutzner, A. (2014). Quality control of oxidatively damaged mitochondrial proteins is mediated by p97 and the proteasome. Free Radic. Biol. Med. 75, 121-128. doi: 10.1016/j.freeradbiomed.2014. 07.016

Karbowski, M., and Neutzner, A. (2012). Neurodegeneration as a consequence of failed mitochondrial maintenance. Acta Neuropathol. 123, 157-171. doi: 10.1007/s00401-011-0921-0

Karbowski, M., Neutzner, A., and Youle, R. J. (2007). The mitochondrial E3 ubiquitin ligase MARCH5 is required for Drpl dependent mitochondrial division. J. Cell Biol. 178, 71-84. doi: 10.1083/jcb.200611064

Kornmann, B. (2014). Quality control in mitochondria: use it, break it, fix it, trash it. F1000Prime Rep. 6:15. doi: 10.12703/P6-15

Leboucher, G. P., Tsai, Y. C., Yang, M., Shaw, K. C., Zhou, M., Veenstra, T. D., et al. (2012). Stress-induced phosphorylation and proteasomal degradation of mitofusin 2 facilitates mitochondrial fragmentation and apoptosis. Mol. Cell 47, 547-557. doi: 10.1016/j.molcel.2012.05.041

Meyer, H., Bug, M., and Bremer, S. (2012). Emerging functions of the VCP/p97 AAA-ATPase in the ubiquitin system. Nat. Cell Biol. 14, 117-123. doi: $10.1038 /$ ncb 2407

Nagashima, S., Tokuyama, T., Yonashiro, R., Inatome, R., and Yanagi, S. (2014). Roles of mitochondrial ubiquitin ligase MITOL/MARCH5 in mitochondrial dynamics and diseases. J. Biochem. 155, 273-279. doi: 10.1093/jb/mvu016

Narendra, D., Tanaka, A., Suen, D. F., and Youle, R. J. (2008). Parkin is recruited selectively to impaired mitochondria and promotes their autophagy. J. Cell Biol. 183, 795-803. doi: 10.1083/jcb.200809125

Narendra, D., Walker, J. E., and Youle, R. (2012). Mitochondrial quality control mediated by PINK1 and Parkin: links to parkinsonism. Cold Spring Harb. Perspect. Biol. 4:a011338. doi: 10.1101/cshperspect.a011338

Neutzner, A., Benard, G., Youle, R. J., and Karbowski, M. (2008). Role of the ubiquitin conjugation system in the maintenance of mitochondrial homeostasis. Ann. N.Y. Acad. Sci. 1147, 242-253. doi: 10.1196/annals.1427.012

Neutzner, A., and Youle, R. J. (2005). Instability of the mitofusin Fzol regulates mitochondrial morphology during the mating response of the yeast Saccharomyces cerevisiae. J. Biol. Chem. 280, 18598-18603. doi: 10.1074/jbc.M500807200

Neutzner, A., Youle, R. J., and Karbowski, M. (2007). Outer mitochondrial membrane protein degradation by the proteasome. Novartis Found. Symp. 287, 4-14; discussion 14-20. doi: 10.1002/9780470725207.ch2

R Core Team. (2013). R: A Language and Environment for Statistical Computing. Vienna: R Foundation for Statistical Computing.

Radi, E., Formichi, P., Battisti, C., and Federico, A. (2014). Apoptosis and oxidative stress in neurodegenerative diseases. J. Alzheimers Dis. 42, S125-S152. doi: 10.3233/JAD-132738

Ruggiano, A., Foresti, O., and Carvalho, P. (2014). Quality control: ER-associated degradation: protein quality control and beyond. J. Cell Biol. 204, 869-879. doi: $10.1083 /$ jcb. 201312042

Schneider, C. A., Rasband, W. S., and Eliceiri, K. W. (2012). NIH Image to Image): 25 years of image analysis. Nat. Methods 9, 671-675. doi: 10.1038/ nmeth.2089 
Sugiura, A., Yonashiro, R., Fukuda, T., Matsushita, N., Nagashima, S., Inatome, R., et al. (2011). A mitochondrial ubiquitin ligase MITOL controls cell toxicity of polyglutamine-expanded protein. Mitochondrion 11, 139-146. doi: 10.1016/j.mito.2010.09.001

Tanaka, A., Cleland, M. M., Xu, S., Narendra, D. P., Suen, D. F., Karbowski, M., et al. (2010). Proteasome and p97 mediate mitophagy and degradation of mitofusins induced by Parkin. J. Cell Biol. 191, 1367-1380. doi: 10.1083/jcb.201 007013

Tang, F., Wang, B., Li, N., Wu, Y., Jia, J., Suo, T., et al. (2011). RNF185, a novel mitochondrial ubiquitin E3 ligase, regulates autophagy through interaction with BNIP1. PLoS ONE 6:e24367. doi: 10.1371/journal.pone. 0024367

Tatsuta, T., and Langer, T. (2008). Quality control of mitochondria: protection against neurodegeneration and ageing. EMBO J. 27, 306-314. doi: 10.1038/sj.emboj.7601972

Wehr, N. B., and Levine, R. L. (2013). Quantification of protein carbonylation. Methods Mol. Biol. 965, 265-281. doi: 10.1007/978-1-62703-239-1_18

Wolf, D. H., and Stolz, A. (2012). The Cdc48 machine in endoplasmic reticulum associated protein degradation. Biochim. Biophys. Acta 1823, 117-124. doi: 10.1016/j.bbamcr.2011.09.002

$\mathrm{Xu}$, S., Peng, G., Wang, Y., Fang, S., and Karbowski, M. (2011). The AAA-ATPase p97 is essential for outer mitochondrial membrane protein turnover. Mol. Biol. Cell 22, 291-300. doi: 10.1091/mbc.E10-09-0748
Yamanaka, K., Sasagawa, Y., and Ogura, T. (2012). Recent advances in p97/VCP/Cdc48 cellular functions. Biochim. Biophys. Acta 1823, 130-137. doi: 10.1016/j.bbamcr.2011.07.001

Youle, R. J., and Narendra, D. P. (2011). Mechanisms of mitophagy. Nat. Rev. Mol. Cell Biol. 12, 9-14. doi: 10.1038/nrm3028

Conflict of Interest Statement: The authors declare that the research was conducted in the absence of any commercial or financial relationships that could be construed as a potential conflict of interest.

Received: 21 July 2014; accepted: 11 January 2015; published online: 02 February 2015. Citation: Fang L, Hemion C, Pinho Ferreira Bento AC, Bippes CC, Flammer $J$ and Neutzner A (2015) Mitochondrial function in neuronal cells depends on p97/VCP/Cdc48-mediated quality control. Front. Cell. Neurosci. 9:16. doi: 10.3389/ fncel.2015.00016

This article was submitted to the journal Frontiers in Cellular Neuroscience. Copyright (C) 2015 Fang, Hemion, Pinho Ferreira Bento, Bippes, Flammer and Neutzner. This is an open-access article distributed under the terms of the Creative Commons Attribution License (CC BY). The use, distribution or reproduction in other forums is permitted, provided the original author(s) or licensor are credited and that the original publication in this journal is cited, in accordance with accepted academic practice. No use, distribution or reproduction is permitted which does not comply with these terms. 